\title{
Tumor-infiltrating lymphocytes as a feasible adjuvant immunotherapy for osteosarcoma with a poor response to neoadjuvant chemotherapy
}

\author{
Jing Shi*, ${ }^{*}$, Ming $\mathrm{Li}^{1} \&$ Rongzhi Yang ${ }^{1}$ \\ ${ }^{1}$ Department of Orthopedic Surgery, Luoyang Central Hospital Affiliated to Zhengzhou University, Luoyang 471009, China \\ *Author for correspondence: sj1980212009@126.com
}

\begin{abstract}
Aim: To investigate the efficacy of adjuvant chemotherapy plus tumor-infiltrating lymphocytes (TILs) therapy in osteosarcoma patients with a poor response to neoadjuvant chemotherapy. Materials \& methods: 40 patients received adjuvant chemotherapy (Group 1) and 40 patients received adjuvant chemotherapy plus TILs therapy (Group 2). Disease-free survival (DFS) and overall survival (OS) were analyzed by KaplanMeier analysis. Results: The median DFS (mDFS; 65.3 months) and median OS (mOS; 95.8 months) in Group 2 were significantly prolonged compared with those in Group 1 ( 55.5 months for mDFS and 80.4 months for mOS). Univariate and multivariate analyses indicated that a greater number of TILs transfused was an independent prognostic factor for both mDFS and mOS. Conclusion: Adjuvant chemotherapy plus TILs therapy may prolong survival of patients with a poor response to neoadjuvant chemotherapy.
\end{abstract}

First draft submitted: 16 April 2020; Accepted for publication: 20 May 2020; Published online: 3 June 2020

Keywords: adjuvant chemotherapy $\bullet$ adverse effects $\bullet$ immunotherapy $\bullet$ osteosarcoma $\bullet$ PD1 $\bullet$ phenotype $\bullet$ poor response $\bullet$ prognostic factor $\bullet$ retrospective $\bullet$ TILs

Osteosarcoma is the most common primary malignant bone tumor, affecting both children and young adults. Recent data have shown that an estimated 3500 people will be diagnosed with osteosarcoma in USA and 1660 patients will die from osteosarcoma every year [1]. The median age of all osteosarcoma patients is $<25$ years [2,3]. Osteosarcoma patients with low-grade tumors only require surgery; however, patients with high-grade tumors require an aggressive treatment regimen [4-9]. The standard treatment regimen of high-grade tumors is neochemotherapy + surgery + adjuvant chemotherapy. A satisfactory prognosis can be achieved from this aggressive treatment regimen in patients in whom the disease has a good response to neochemotherapy. In patients in whom the disease has a poor response to neochemotherapy, attempts to improve the outcome by modifying the adjuvant chemotherapy regimen have been unsuccessful [9-11].

Currently, most patients with high-grade tumors require neoadjuvant chemotherapy, followed by surgical excision of the tumor with a wide margin of healthy tissues around the tumor. After surgery, chemotherapy drugs are chosen for adjuvant therapy based on the necrosis rate $[7,8,10,11]$. A good histopathologic response to neoadjuvant chemotherapy has been shown to be predictive for overall survival (OS), independent of the chemotherapy regimen administered after surgery $[10,12,13]$. Although previous studies have attempted to improve outcomes of patients with osteosarcoma by modifying the dose intensity of chemotherapy agents or adding new chemotherapy agents, such attempts have been unsuccessful in osteosarcoma patients with a poor response to neoadjuvant chemotherapy [14-17]. Therefore, the identification of new methods of adjuvant therapy for osteosarcoma patients is urgently needed.

Recently, immunotherapy, such as vaccines, adoptive cellular therapies and checkpoint inhibitors, has emerged as a treatment method for many cancer types [18-21]. Interestingly, many studies suggest that tumor-infiltrating lymphocytes (TILs) get exhausted in the osteosarcoma microenvironment, which is one of the significant reasons for osteosarcoma recurrence [22-24]. TILs therapy has achieved satisfactory treatment effects for metastatic melanoma patients with a reported objective response rate (ORR) of $40-70 \%[20,25,26]$. It is still unclear, however, whether

Future Medicine 
TILs therapy can achieve a satisfactory treatment effect for osteosarcoma. In a preclinical study, TILs extracted from osteosarcomas penetrate the tumor microenvironment and have cytotoxic effects against allogeneic tumor cells, which suggested that TILs therapy could be a very efficient strategy for the treatment of osteosarcoma [27]. Therefore, TILs therapy may be a very efficient strategy for the treatment of osteosarcoma.

In this study, we analyzed the efficacy of combined chemotherapy with TILs therapy among osteosarcoma patients with a poor response to neoadjuvant chemotherapy. Our results suggest that TILs therapy combined with adjuvant chemotherapy improves the median disease-free survival (mDFS) and median overall survival (mOS) of osteosarcoma patients with a poor response to neoadjuvant chemotherapy.

\section{Materials \& methods}

\section{Patients selection}

We performed a retrospective study to evaluate the clinical outcomes of TILs therapy for patients with primary high-grade intramedullary osteosarcoma. A total of 80 osteosarcoma patients with poor responses to neoadjuvant chemotherapy between 1 January 2011 and 1 January 2014 were enrolled in this study. The enrolled criteria were as follows: all the enrolled patients were first diagnosed with osteosarcoma at our hospital and all the enrolled patients had primary high-grade intramedullary osteosarcoma and received neochemotherapy before surgery; all the enrolled patients were required to have a diagnosis of histopathologic response to neochemotherapy. Response was defined by pathologic mapping per institutional guidelines, as previous described [9]. In patients with a good response, the amount of viable tumor was $<10 \%$ of the tumor area. In patients with a poor response, the amount of viable tumor was $>10 \%$ of the tumor area; only patients with poor responses to neoadjuvant chemotherapy were collected in this study; CT scans of the chest, abdomen and bone were required to confirm no metastases at the beginning of neochemotherapy; patients had to be $>11$ of age; normal blood parameters, renal function, hepatic function and left ventricular function were necessary; a Southwest Oncology Group (SWOG) performance status of 0-2 was required. The exclusion criteria were as follows: multicentric primary diseases, such as heart disease, hepatitis and renal insufficiency; pregnant or nursing women; immune-related diseases; and relapsed or/and metastatic diseases at presentation. The present study was approved by the Ethics Committee at our institution and an approved consent form was signed by all the patients.

\section{Study design \& follow-up}

This single-center retrospective clinical study was approved by the Ethics Committee at the Affiliated Luoyang Central Hospital of Zhengzhou University. All methods and procedures associated with this study were conducted in accordance with the Good Clinical Practice guidelines and accorded ethically with the principles of the Declaration of Helsinki and local laws. All authors had access to the study data and reviewed and approved the final manuscript. The primary end point was DFS time, with DFS rates measured at 5-year intervals. The secondary end points were 5-year OS time rates, as well as OS as evaluated by Kaplan-Meier analysis. Potential prognostic factors were also analyzed by univariate and multivariate analyses based on adjuvant chemotherapy plus TILs therapy. The DFS was calculated from the date of surgery to the time of recurrence or metastasis. Patients free of these events were censored at the time of last contact. The OS was calculated from the date of surgery to the time of death, and patients who were alive at the time of last contact were censored. DFS and OS were calculated by the Kaplan-Meier method. After neoadjuvant chemotherapy, all patients underwent wide excision of primary tumors. The pathologic evaluation indicated that all patients had negative margins with a poor response. A total of 90 patients were screened, 50 of whom agreed with adjuvant chemotherapy plus TILs therapy, 40 of whom agreed with adjuvant chemotherapy, 10 of whom were excluded due to failure of TILs expansion. Therefore, after surgery, 80 patients were assigned to Group $1(n=40$; adjuvant chemotherapy) or Group 2 ( $n=40$; adjuvant chemotherapy plus TILs therapy). All patients in Group 2 received one cycle of TIL transfusion at the time point of after surgery. Neochemotherapy and adjuvant chemotherapy regimen in the two groups were the same. High-dose methotrexate, cisplatin and doxorubicin (MAP), which is the preferred regimen, was chosen for neochemotherapy and adjuvant chemotherapy. The chemotherapy regimen conformed to the $2010 \mathrm{NCCN}$ clinical Practice Guidelines in Oncology. After adjuvant therapy, all the patients were scheduled for follow-up evaluations at our hospital from the date of initial treatment to the follow-up deadline, 8 January 2020, or the time of death. Clinical examinations were performed by our oncology specialists every 3 months, including complete blood examinations, chest and abdominal CT scans and surgery area MRI scan during the first 2-year period. During years 2-5, patients were examined every 6 months. Beyond 5 years, patients were examined every year. If follow-up evaluations showed 
metastatic disease and/or local recurrence, other therapies were applied, including conventional therapies (surgery, chemotherapy and radiotherapy) and immunotherapy.

\section{Obtained primary tumor specimens \& generation of TILs}

The culture method of TILs from each patient has been described in detail in previous studies $[20,25,27,28]$. Briefly, the primary osteosarcoma specimens with less calcification (the diameter is approximately $3 \pm 0.6 \mathrm{~cm}$ ) from patients were obtained from surgically resected tumors and TILs were cultured as follows: tumor tissues (softer tissues examined by two pathologists, which are easy to slice) were sliced into pieces $2-3 \mathrm{~mm}^{3}$ in size using a scalpel [27]; collagenase type IV (1 mg/ml; Sigma-Aldrich, MO, USA), DNase I (2 U/ml; Sigma-Aldrich, MO, USA) and hyaluronidase type $\mathrm{V}(0.5 \mathrm{U} / \mathrm{ml}$; Sigma-Aldrich, MO, USA) were used to perform enzymatic digestion of the tissues for approximately $3 \mathrm{~h}$ at room temperature to obtain single-cell suspensions; single-cell suspensions were filtered, washed twice with phosphate-buffered saline (PBS), counted the cell numbers (the cell numbers are approximately $2-3 \times 10^{6}$ per patient) and then incubated in a 12 -well plate at a concentration of $1.0 \times 10^{6}$ TILs/ml in X-VIVO medium (Muenchensteinerstrasse $38 \mathrm{CH}-4002$ Basel, Switzerland) with $7000 \mathrm{IU} / \mathrm{ml}$ of recombinant human IL-2 (rhIL-2, Novartis, UK), considered day 0 ; on day 1 , the cell suspensions were removed and further purified through a Ficoll gradient, the purified bulk TIL culture was maintained at a concentration of $1-2 \times 10^{6}$ cells $/ \mathrm{ml}$ in X-VIVO medium with $7000 \mathrm{IU} / \mathrm{ml}$ of rhIL-2 until all other cells (including tumor cells) were eliminated and a cell number of at least $5 \times 10^{7}$ TIL cells was achieved, and this culture process required 10-14 days; and the cultured TIL cells were immediately used with anti-CD3 antibody $(30 \mathrm{ng} / \mathrm{ml}$; GE Healthcare Biosciences, PA, USA) and $1000 \mathrm{IU} / \mathrm{ml}$ of rhIL-2 for large-scale expansion and this culture process required 3-5 days. By this process, cultures were expanded to at least $3 \times 10^{9}$ TILs. After counting the cell numbers, TILs were harvested. After detecting the immunophenotyping of TILs and confirming that the cell suspensions were free of bacterial and fungal contamination, negative for Mycoplasma, and contained $<5$ Eu endotoxin, TILs were infused back into patients.

\section{Phenotype assessment of TILs by flowcytometry}

To determine the quality of cultured TILs. The phenotypes of freshly isolated and cultured TILs were identified by flow cytometry. The antibodies used in this study were purchased from BD Biosciences and the detailed information were described as follows. The flowcytometry method followed published descriptions [28]. Specifically, $1 \times 10^{6}$ freshly isolated or cultured TILs were blocked with blocking buffer (PBS $+1 \%$ BSA $+0.4 \%$ human serum), then stained with anti-CD3 (Cat\#: 555339, 2ul/106 cells), CD4(Cat\#: 557871, 3ul/10 cells), CD8(Cat\#: 563823, $2 \mathrm{ul} / 10^{6}$ cells), CD16(Cat\#: $563829,2 \mathrm{ul} / 10^{6}$ cells) and CD56 antibodies (Cat\#: 557919 , 3ul/10 cells) in the dark on ice for $30 \mathrm{~min}$. The cells were washed in PBS one time, resuspended in $300 \mathrm{ul}$ of PBS, and run on a Fortessa cell analyzer (BD Bioscience). Fluorescence minus one was considered to be the negative control. Flowjo software (BD Bioscience) was used to analyze flow cytometry data. To detect the expression of PD1 on $\mathrm{CD} 3^{+} \mathrm{CD} 8^{+}$fresh TILs in the primary tumor specimens and analyze the correlation between PD1 expression and mDFS and mOS, mouse anti-human CD279 antibody (PD1, Cat\#: 561272, 5ul/106 cells; BD Bioscience) was used to detect the expression of $\mathrm{PD} 1$ on $\mathrm{CD}^{+} \mathrm{CD}^{+}$fresh TILs from both Groups. The same flowcytometry method was used.

\section{Statistical analysis}

GraphPad Prism 8.0 and SPSS17.0 software was used for statistical analyses. DFS and OS were calculated by the Kaplan-Meier method from the time of surgery. The prognostic factors were analyzed by univariate and multivariable Cox proportional hazards regression models. The $\chi 2$ test was used in a prespecified analysis to compare the characteristics of Groups. Other data were analyzed using a t-test. For all statistical analyses, significance was indicated at a $\mathrm{p}<0.05$ level.

\section{Results}

\section{Patient characteristics}

Between 1 January 2011 and 1 January 2014, 80 osteosarcoma patients with poor response to neoadjuvant chemotherapy after surgery were enrolled in this study. Forty patients received adjuvant chemotherapy alone (Group 1) and 40 patients received adjuvant chemotherapy plus TILs therapy (Group 2). The baseline characteristics of patients in the two groups are detailed in Table 1; With the exception of adjuvant therapy after surgery, there were no significant differences between the two groups at presentation and treatment regimens after metastases (Table 1). 
Table 1. The detailed baseline of the 80 patients.

\begin{tabular}{|c|c|c|c|c|}
\hline Characteristics & Group1 $(n=40)$ & Group $2(n=40)$ & $x^{2}$ & p-value \\
\hline \multicolumn{5}{|l|}{ Age(years) } \\
\hline$<25$ & 34 & 33 & & \\
\hline$\geq 25$ & 6 & 7 & 0.092 & 0.762 \\
\hline \multicolumn{5}{|l|}{ Gender } \\
\hline Male & 30 & 25 & & \\
\hline Female & 10 & 15 & 1.455 & 0.228 \\
\hline \multicolumn{5}{|c|}{ Location of primary tumor } \\
\hline Femur and tibia & 33 & 32 & & \\
\hline Others & 7 & 8 & 0.082 & 0.775 \\
\hline \multicolumn{5}{|c|}{ Size of primary tumor $(\mathrm{cm})$} \\
\hline$\geq 5$ & 24 & 30 & & \\
\hline$<5$ & 16 & 10 & 2.051 & 0.152 \\
\hline \multicolumn{5}{|c|}{ Histological classification } \\
\hline Conventional & 35 & 37 & & \\
\hline others & 5 & 3 & 0.556 & 0.456 \\
\hline \multicolumn{5}{|c|}{ Metastatic sites after adjuvant therapy } \\
\hline Lung & 38 & 36 & & \\
\hline Others & 2 & 4 & 0.241 & 0.623 \\
\hline \multicolumn{5}{|c|}{ Treatments after metastases } \\
\hline Surgery & 15 & 18 & & \\
\hline Chemotherapy & 40 & 40 & & \\
\hline Radiotherapy & 10 & 8 & & \\
\hline Immunotherapy & 25 & 28 & 0.578 & 0.901 \\
\hline
\end{tabular}

Phenotypic analysis of freshly isolated \& cultured TILs

We identified the phenotypes of freshly isolated TILs and cultured TILs by flow cytometry. Figure 1A indicates the representative flow cytometry analysis of $\mathrm{CD} 3^{+}, \mathrm{CD}^{+} \mathrm{CD}^{+}, \mathrm{CD}^{+} \mathrm{CD} 8^{+}, \mathrm{CD} 3^{+} \mathrm{CD} 56^{+}$and $\mathrm{CD} 3^{-} \mathrm{CD} 16^{+} \mathrm{CD} 56^{+}$populations of TILs before and after ex vivo cultures. Quantitative analysis demonstrated that the percentages of $\mathrm{CD}^{+}, \mathrm{CD}^{+} \mathrm{CD}^{+}, \mathrm{CD}^{+} \mathrm{CD} 8^{+}$and $\mathrm{CD} 3^{+} \mathrm{CD} 56^{+}$increased significantly $(\mathrm{p}<0.01)$; however, the percentage of $\mathrm{CD} 3^{-} \mathrm{CD} 16^{+} \mathrm{CD} 56^{+}$cells decreased significantly $(\mathrm{p}<0.01)$ (Figure 1B).

\section{Treatment outcomes}

By using this type of cultured TILs, we assigned 80 patients into the two groups, Group 1 received adjuvant chemotherapy and Group 2 received adjuvant chemotherapy plus TILs immunotherapy. The date of the last follow-up visit was 8 January 2020. Of the 80 eligible patients, 56 died by the last follow-up evaluation. As shown in Figure $2 \mathrm{~A} \& \mathrm{~B}$, the mDFS and mOS of Groups 1 and 2 were 55.5 months and 65.3 months $(\mathrm{p}<0.0001)$, and 80.4 months and 95.8 months $(\mathrm{p}<0.0001)$, respectively. Then, we analyzed the 5-year DFS and 5-year OS rates. Our results indicated that the 5-year DFS and 5-year OS rates in Groups 1 and 2 were 50 and $72.5 \%(\mathrm{p}<0.0001)$, and $65 \%$ and $80 \%(\mathrm{p}<0.0001)$, respectively. Taken together, our data showed that osteosarcoma patients with a poor response to neoadjuvant chemotherapy benefit from adjuvant chemotherapy plus TILs therapy compared with adjuvant chemotherapy alone.

\section{Prognostic factors of patients in Group 2}

Having shown that the mDFS and mOS were increased significantly in Group 2, we then examined potential prognostic factors in Group 2. We analyzed a number of factors, such as gender, age, tumor site, tumor size, histologic classification, metastatic sites after adjuvant therapy and the number of TILs infused. As summarized in Table 2, univariate analyses proposed that a greater number of TILs infused was significantly associated with increased mDFS (69.8 and 56.9 months; $\mathrm{p}<0.0001)$ and $\mathrm{mOS}$ (103.1 and 83.05 months; $\mathrm{p}=0.0003)$. Moreover, multivariate analyses (Tables $3 \& 4$ ) suggested that a greater number of TILs infused was an independent prognostic element in osteosarcoma patients with adjuvant chemotherapy plus TILs therapy for both $\mathrm{mDFS}$ and $\mathrm{mOS}$ (hazard ratio $[\mathrm{HR}]=3.15 ; \mathrm{p}=0.0001$; and $\mathrm{HR}=2.8 ; \mathrm{p}=0.003$, respectively). Although, univariate analyses showed that patients with $<25$ years of age had a longer mDFS compared with patients $>25$ years of age, while there was no significant difference of mOS. Importantly, multivariate analyses indicate that there is no significant difference based on age. Collectively, our data implied that a greater number of TILs infused was a potential significant prognostic factor in osteosarcoma patients with adjuvant chemotherapy plus TILs therapy. 


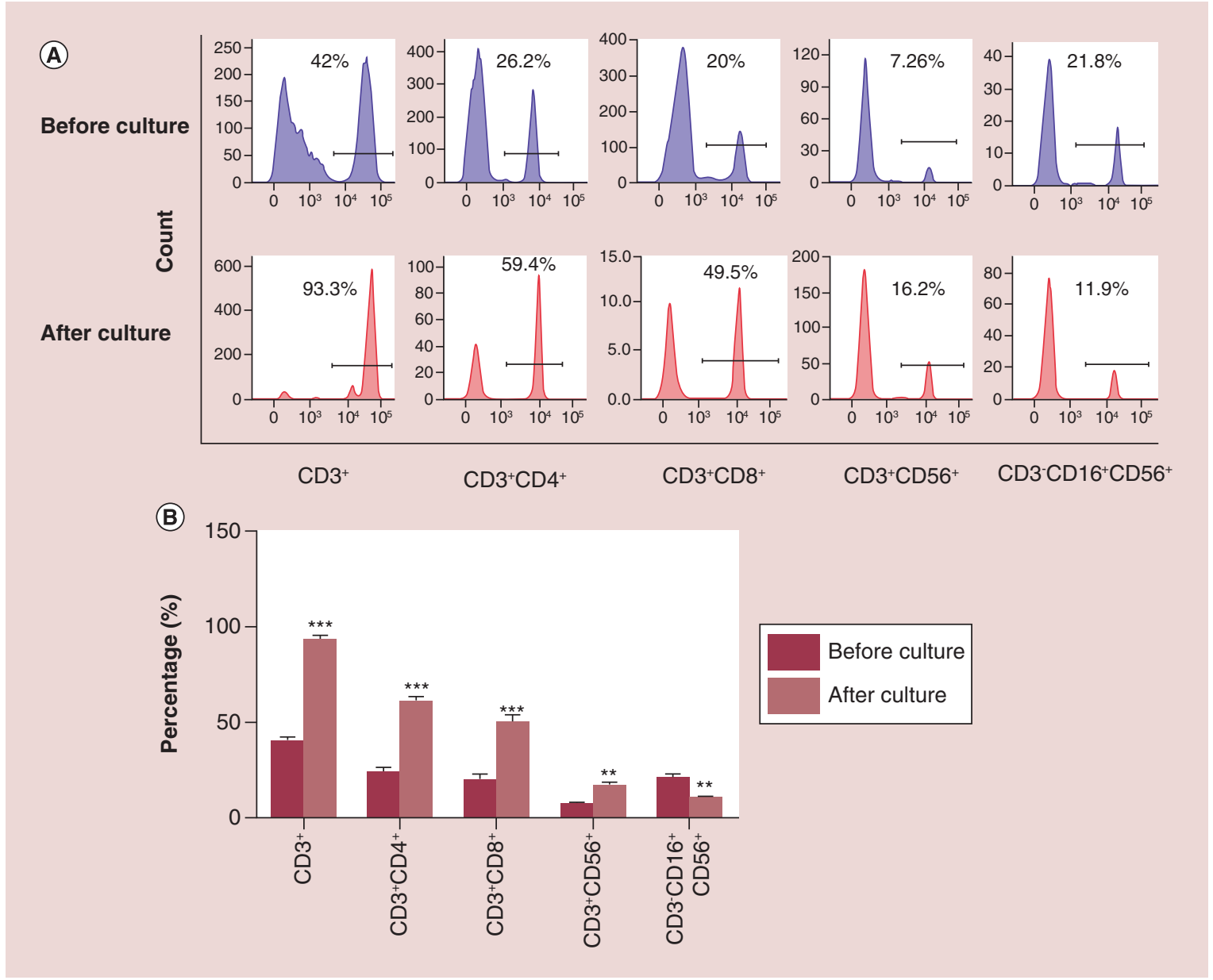

Figure 1. Phenotypic analysis of freshly isolated and cultured tumor-infiltrating lymphocytes. (A) Representative flow cytometry of $\mathrm{CD}^{+}, \mathrm{CD}^{+} \mathrm{CD}^{+}, \mathrm{CD}^{+} \mathrm{CD}^{+}, \mathrm{CD}^{+} \mathrm{CD}^{+} 6^{+}, \mathrm{CD}^{-} \mathrm{CD} 16^{+} \mathrm{CD}^{2} 6^{+}$percentage of TILs from freshly isolated and cultured in vitro. (B) Quantitative analysis of $\mathrm{CD}^{+}, \mathrm{CD}^{+} \mathrm{CD} 4^{+}, \mathrm{CD}^{+} \mathrm{CD} 8^{+}, \mathrm{CD}^{+} \mathrm{CD}^{+} 6^{+}$, $\mathrm{CD}^{-} \mathrm{CD} 16^{+} \mathrm{CD}^{+} 6^{+}$percentage of TILs from freshly isolated and cultured in vitro.

TIL: Tumor-infiltrating lymphocyte.

(A)

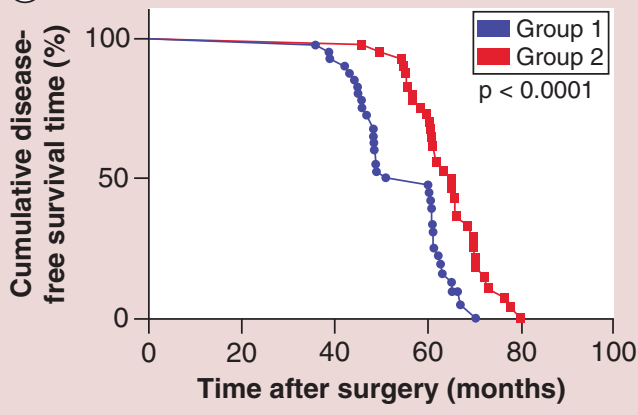

(B)

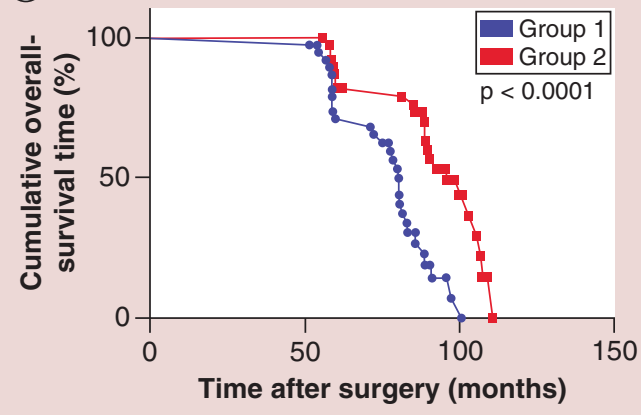

Figure 2. Kaplan-Meier curves for disease-free survival and overall survival of osteosarcoma patients with a poor response to neoadjuvant chemotherapy according to Group 1 (adjuvant chemotherapy) and Group 2 (adjuvant chemotherapy plus tumor-infiltrating lymphocytes therapy), $\mathbf{n}=\mathbf{8 0}$. (A) The DFS curves based on Group 1 (adjuvant chemotherapy) and Group 2 (adjuvant chemotherapy plus TILs therapy). (B) The OS curves based on Group 1 (adjuvant chemotherapy) and Group 2 (adjuvant chemotherapy plus TILs therapy). DFS: Disease-free survival; OS: Overall survival; TIL: Tumor-infiltrating lymphocyte. 
Table 2. Univariate analysis of factors related to median disease-free survival and median overall survival of Group 2 patients in this study $(n=40)$.

\begin{tabular}{|c|c|c|c|c|}
\hline Characteristics & mDFS (months) & p-value & mOS (months) & p-value \\
\hline \multicolumn{5}{|l|}{ Age (years) } \\
\hline$<25$ & 65.9 & & 99.7 & \\
\hline$\geq 25$ & 60.5 & 0.019 & 88.9 & 0.4026 \\
\hline \multicolumn{5}{|l|}{ Gender } \\
\hline Male & 63.5 & & 92.6 & \\
\hline Female & 68.7 & 0.2652 & 105.6 & 0.2672 \\
\hline \multicolumn{5}{|c|}{ Location of primary tumor } \\
\hline Femur and Tibia & 65.4 & & 95.8 & \\
\hline Other & 63.15 & 0.5929 & 110.7 & 0.4413 \\
\hline \multicolumn{5}{|c|}{ Size of primary tumor $(\mathrm{cm})$} \\
\hline$\geq 5$ & 63.5 & & 92.6 & \\
\hline$<5$ & 67.55 & 0.2845 & 103.1 & 0.4137 \\
\hline \multicolumn{5}{|c|}{ Histological classification } \\
\hline Conventional & 73.1 & & 110.7 & \\
\hline others & 61.8 & 0.190 & 92.6 & 0.154 \\
\hline Lung & 65.9 & & 99.7 & \\
\hline Others & 61.8 & 0.6152 & 92.6 & 0.7989 \\
\hline \multicolumn{5}{|c|}{ Number of TILs infused } \\
\hline$\geq 5 \times 10^{9}$ & 69.8 & & 103.1 & \\
\hline$<5 \times 10^{9}$ & 56.9 & $<0.0001$ & 83.05 & 0.0003 \\
\hline
\end{tabular}

\begin{tabular}{|c|c|c|c|}
\hline Parameters & Hazard ratio & $95 \%$ confidence interval & p-value \\
\hline $\begin{array}{l}\text { Age (years) ( }<25 \text { vs } \geq 25 \text { ) } \\
\text { number of TILs infused }\end{array}$ & 0.835 & $(0.782,0.989)$ & 0.152 \\
\hline$\left(\geq 5 \times 10^{9}\right.$ vs $\left.<5 \times 10^{9}\right)$ & 3.15 & $(1.51,5.88)$ & 0.0001 \\
\hline
\end{tabular}

\begin{tabular}{|c|c|c|c|}
\hline Parameters & Hazard ratio & $95 \%$ confidence interval & p-value \\
\hline $\begin{array}{l}\text { Age (years) }(<25 \text { vs } \geq 25) \\
\text { number of TILs infused } \\
\left(\geq 5 \times 10^{9} \text { vs }<5 \times 10^{9}\right)\end{array}$ & 0.52 & $(0.49,0.67)$ & 0.51 \\
\hline
\end{tabular}

PD1 expression by $\mathrm{CD}^{+}{ }^{+} \mathrm{CD} 8^{+}$TILs showed different prognostic effects between Groups 1 \& 2

PD1 is expressed by T cells and can be induced by tumors, but become exhausted in the microenvironment, which is one of the main reasons for osteosarcoma recurrence. We then examined PD1 expression by $\mathrm{CD} 3^{+} \mathrm{CD} 8^{+} \mathrm{TILs}$ from tumor specimens and analyzed the correlation between $\mathrm{CD} 3^{+} \mathrm{CD} 8^{+} \mathrm{PD} 1^{+} \mathrm{TILs}$ with prognosis of osteosarcoma in both Groups 1 and 2 . The percentages of $\mathrm{CD}^{+}{ }^{+} \mathrm{CD} 8{ }^{+} \mathrm{PD} 1^{+}$TILs in Groups 1 and 2 were $15.13 \% \pm 3.56 \%$ $(\mathrm{n}=40)$ and $14.62 \% \pm 4.35 \%(\mathrm{n}=40)$, respectively. There were no significant differences between Groups 1 and 2 of PD1 expression on $\mathrm{CD} 3^{+} \mathrm{CD} 8{ }^{+}$TILs. Based on the expression of PD1 on $\mathrm{CD} 3^{+} \mathrm{CD} 8{ }^{+}$TILs, we divided patients into PD $1^{\text {hi }}(\geq 10 \%)$ and PD $1^{\text {low }}(<10 \%)$ in Groups 1 and 2 . Consistent with previous studies, PD $1^{\text {hi }}$ had a decreased mDFS (48.0 vs 61.2 months; $\mathrm{p}=0.0005)$ and mOS (65.0 vs 82.9 months; $\mathrm{p}=0.0018)$ compared with PD $1^{\text {low }}$ in Group 1 (Figure 3A \& B). In contrast, PD $1^{\text {hi }}$ had an increased mDFS (73.1 vs 61.0 months; $\mathrm{p}<0.0001$ ) and mOS (107.3 vs 88.9 months; $\mathrm{p}<0.0001$ ) compared with PD $1^{\text {low }}$ in Group 2 (Figure $3 \mathrm{C} \& \mathrm{D}$ ). Interestingly, multivariate analyses suggested that $\mathrm{PD} 1{ }^{\mathrm{hi}}$ was an independent poor prognostic element in osteosarcoma patients for $\mathrm{mDFS}$ and $\mathrm{mOS}$ ( $\mathrm{HR}=2.4 ; \mathrm{p}=0.0003$ and $\mathrm{HR}=3.5 ; \mathrm{p}<0.0001$, respectively) in Group 1. In contrast, $\mathrm{PD} 1{ }^{\mathrm{hi}}$ was an independent good prognostic element in osteosarcoma patients for $\mathrm{mDFS}$ and $\mathrm{mOS}(\mathrm{HR}=3.3$; $\mathrm{p}$ $<0.0001$ and $\mathrm{HR}=4.1 ; \mathrm{p}<0.0001$, respectively) in Group 2. Taken together, our results demonstrate that PD $1^{\text {hi }}$ is a poor prognostic factor in Group1, but a good prognostic factor in Group 2. 
(A)

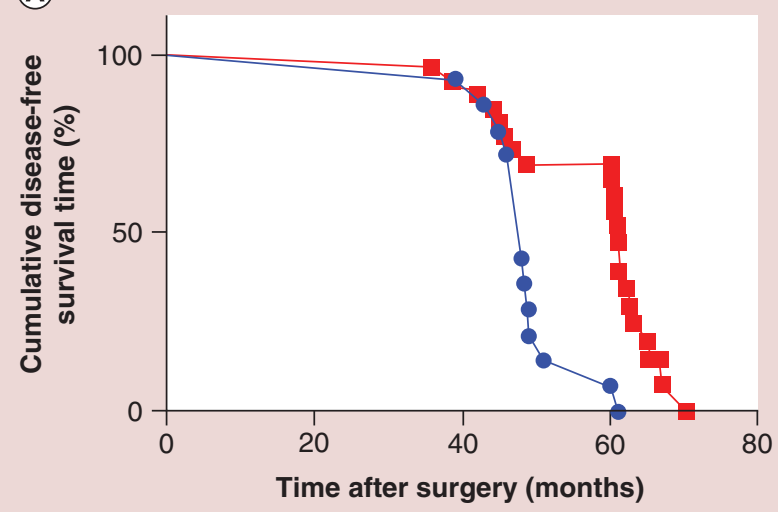

(C)

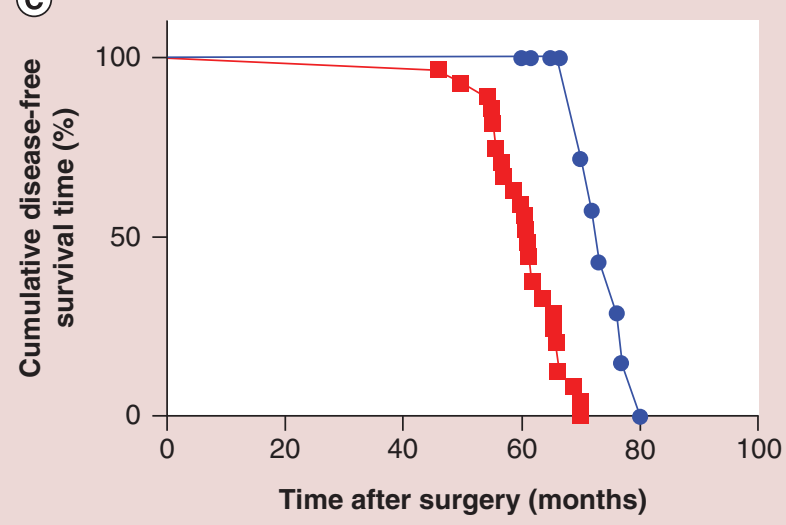

(B)

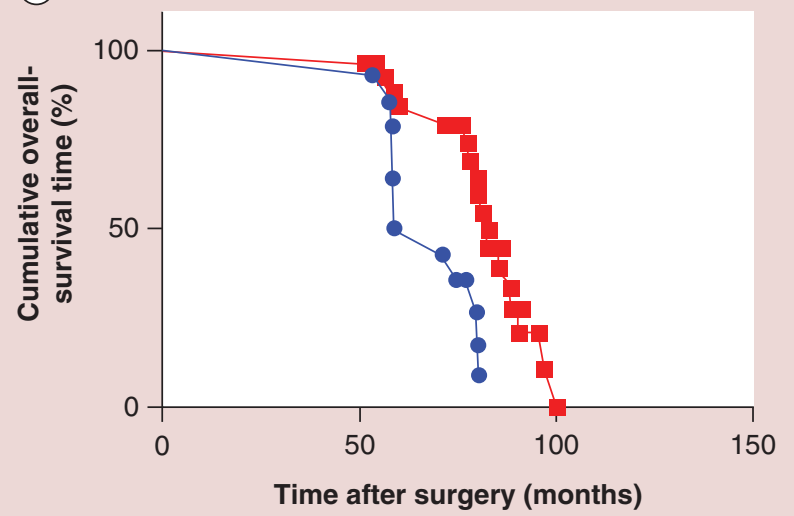

(D)

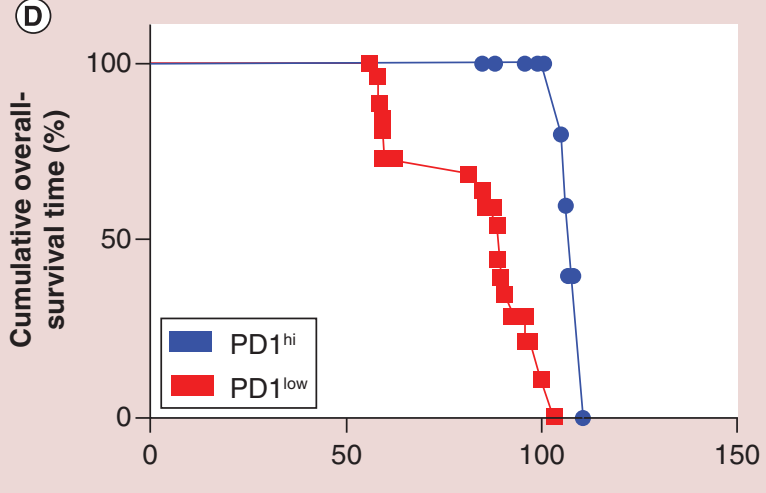

Time after surgery (months)

Figure 3. Kaplan-Meier curves for disease-free survival and overall survival of osteosarcoma patients with poor response to neoadjuvant chemotherapy according to PD1 ${ }^{\text {hi }}$ and PD1 ${ }^{\text {low }}$ in Group 1 (adjuvant chemotherapy) and Group 2 (adjuvant chemotherapy plus tumor-infiltrating lymphocytes therapy), $\mathbf{n}=\mathbf{8 0}$. (A) The DFS curves based on PD $1^{\text {hi }}$ and PD1 low in Group 1 (adjuvant chemotherapy). (B) The OS curves based on PD $1^{\text {hi }}$ and PD $1^{\text {low }}$ in Group 1 (adjuvant chemotherapy). (C) The DFS curves based on PD $1^{\text {hi }}$ and PD $1^{\text {low }}$ in Group 2 (adjuvant chemotherapy plus TILs therapy). (D) The OS curves based on PD1 ${ }^{\text {hi }}$ and PD1 low in Group 2 (adjuvant chemotherapy plus TILs therapy).

DFS: Disease-free survival; OS: Overall survival; TIL: Tumor-infiltrating lymphocyte.

Table 5. Distribution of adverse events.

\begin{tabular}{|c|c|c|c|c|}
\hline \multirow[t]{2}{*}{ Sides effects } & \multicolumn{2}{|c|}{ Group 1} & \multicolumn{2}{|c|}{ Group 2} \\
\hline & Grade 1/2 & Grade $3 / 4$ & Grade $1 / 2$ & Grade $3 / 4$ \\
\hline Fever & 13 & 0 & 30 & 0 \\
\hline Arthralgia & 15 & 0 & 18 & 0 \\
\hline Nausea & 27 & 0 & 29 & 0 \\
\hline Leukopenia & 25 & 10 & 26 & 9 \\
\hline Liver dysfunction & 7 & 5 & 9 & 6 \\
\hline Anemia & 18 & 8 & 16 & 7 \\
\hline Vitiligo & 0 & 0 & 0 & 0 \\
\hline
\end{tabular}

\section{Adverse effects}

In this study, all the patients completed TILs therapy. There were no severe adverse effects (Grade 3 or 4) associated with TILs therapy. There were no significant differences between the two groups with respect to adverse effects (Table 5). 30 patients in the adjuvant chemotherapy plus TILs therapy group developed fevers, the body temperature did not exceed $38.5^{\circ} \mathrm{C}$ and the fever resolved spontaneously within $8 \mathrm{~h}$ in all patients. These data showed that 
adjuvant chemotherapy plus TILs therapy did not increase adverse effects compared with adjuvant chemotherapy alone.

\section{Discussion}

Surgery remains an essential treatment method for patients with osteosarcoma. Although adjuvant chemotherapy clearly improves OS in osteosarcoma patients, the value of continuing to increase dose intensity or add nonspecific cytotoxic agents seems limited for patients with a poor response to neoadjuvant chemotherapy [14-17]. Additionally, in an analysis of 881 patients with nonmetastatic osteosarcoma treated with neoadjuvant chemotherapy and surgery, Bacci et al. showed that the 5-year DFS and OS in good and poor responders were 67.9 and $51.3 \%(\mathrm{p}<0.001)$ and 78.4 and $63.7 \%(\mathrm{p}<0.001)$, respectively [11]. Another report from the Children's Oncology and Group showed similar results [10]. These two studies indicate that patients with a poor response to neoadjuvant chemotherapy had decreased OS. Therefore, several studies attempted to prolong OS of poor responders by modifying the adjuvant chemotherapy; however, were unsuccessful [14-17]. There has been a lack of improvement in survival rates during the past decades. Currently, greater than a half of the patients within an unselected OS population eventually succumb to the disease, despite the current multimodal primary treatments, as well as second-line chemotherapy and surgical metastasectomy [29]. Interestingly, adjuvant chemotherapy with high-dose ifosphamide plus etoposide can result in secondary leukemia in some cases, and addition of the immunotherapeutic mifamuride, to the three-drug regimen, which resulted in a reduction in mortality and an increase in the 8-year survival rate from 70-78\% [30]. The success of mifamurtide in patients indicates that osteosarcoma is sensitive to immunotherapy, suggesting that immunotherapy may be a useful treatment regimen for osteosarcoma. In this study, we found that adjuvant chemotherapy plus TIL immunotherapy significantly increased the mDFS and mOS of osteosarcoma patients with a poor response to neoadjuvant chemotherapy.

Adoptive cell therapy of TILs has achieved a satisfactory treatment effect for metastatic melanoma patients with reported ORR of $40-70 \%$ for several years $[20,25,26]$. Although, TILs also represent an exciting therapeutic approach in numerous other malignant tumors, such as breast and cervical cancer [31-34]; however, there are no reports involving osteosarcoma in clinical use. In a preclinical study, TILs extracted from osteosarcoma can penetrate the tumor microenvironment and have cytotoxic effects against allogeneic tumor cells, which demonstrated that TILs therapy is a very efficient strategy for the treatment of osteosarcoma [27]. Another preclinical study also indicated that epidermal growth factor receptor (HER-2)-specific T cells target drug-resistant tumor-initiating cells in established osteosarcoma cell lines, suggesting that incorporating immunotherapy into current treatment strategies for osteosarcoma has the potential to improve outcomes [35]. Recent studies indicate that chemotherapy agents selectively target highly proliferative cells and hence preferentially destroy the immunosuppressive cells that comprise the microenvironment of growing tumors [36,37]. Therefore, combined chemotherapy with immunotherapy may be a potential treatment method for osteosarcoma patients. Indeed, in the previous study, Rosenberg et al. used TILs combined with nonmyeloablative chemotherapy to treat metastatic melanoma and achieved satisfactory ORRs [25]. In our study, we combined adjuvant chemotherapy with TILs therapy to treat osteosarcoma patients with a poor response to neoadjuvant chemotherapy. By using this method, we balance immune cell populations toward tumor suppression, thus further enhancing the immunoregulatory effect of chemotherapy. In a murine model, lymphodepletion by chemotherapy or chemoradiation seemed to enhance the antitumor effects of transferred T cells in vivo by several mechanisms including the elimination of suppressive $\mathrm{CD} 4{ }^{+} \mathrm{CD} 25^{+} \mathrm{T}$-regulatory lymphocytes, the elimination of cellular 'sinks' for homeostatic cytokines such as IL-7 and IL-15, and the engagement of Toll-like receptors on antigen-presenting cells after damage to the integrity of the gut epithelial lining [38]. In clinic, Steven A Rosenberg's group indicated that host lymphodepletion using chemotherapy or chemoradiation preparative regimens followed by autologous TIL transfer and IL-2 results in objective response rates of $50-70 \%$ in patients with metastatic melanoma refractory to standard therapies [26]. Therefore, it is no surprise that combined chemotherapy with immunotherapy exhibit a synergistic and reciprocal increase in efficacy. We demonstrate that incorporating TILs immunotherapy into adjuvant chemotherapy for osteosarcoma patients with a poor response to neoadjuvant chemotherapy has the potential to improve outcomes. In the future, more studies should be conducted to investigate the clinical efficacy of combined adjuvant chemotherapy with TILs therapy in the management of osteosarcoma.

To explore the prognostic factors governing the efficacy of TILs therapy, we analyzed individual results and correlated factors to gender, age, tumor site, tumor size, metastatic sites after adjuvant therapy and the number of TILs infused. Univariate and multivariate analyses identified the number of TILs infused as a potential predictive 
factor. Many studies have indicated that tumor site and size, age, location of metastases, histologic response to chemotherapy and type of surgery are significant prognostic factors for patients with osteosarcoma [39-41]. In this study, we only enrolled patients with a poor response to neoadjuvant chemotherapy and all of the patients had the same type of surgery. Importantly, we attempted to identify the prognostic factors based on adjuvant chemotherapy plus TILs therapy. Our results suggest that osteosarcoma patients with a poor response to neoadjuvant chemotherapy benefit from adjuvant chemotherapy plus TILs therapy and a greater number of TILs infused is beneficial.

Another interesting finding was the effect of PD1 expression on $\mathrm{CD}^{+}{ }^{+} \mathrm{CD} 8^{+}$TILs in the tumor microenvironment in different treatment groups. Consistent with previous studies, $\mathrm{PD} 1^{\text {hi }}$ is a poor prognostic factor in the adjuvant chemotherapy group; however, $\mathrm{PD} 1^{\mathrm{hi}}$ is a good prognostic factor in adjuvant chemotherapy plus TILs therapy group. Previous studies have shown that PD1 is an inhibitory receptor and CD8 ${ }^{+} \mathrm{PD} 1^{+}$TILs are functionally impaired [42]. Fresh $\mathrm{CD} 8^{+} \mathrm{PD} 1^{+}$TILs cannot make IFN- $\gamma$ by stimulation with PMA-ionomycin in vitro [43]. Therefore, $\mathrm{PD} 1^{\text {hi }}$ is a poor prognostic factor in many malignant tumors, including osteosarcoma; however, Rosenberg et al. reported that purified populations of $\mathrm{CD} 8^{+} \mathrm{PD} 1^{+} \mathrm{TIL}$ s regain function during in vitro expansion in IL-2 and make IFN- $\gamma$ on stimulation with autologous or HLA matched tumor cells [43]. This finding is consistent with clinical results in which the transfusion of cultured TILs have tumor reactivity after in vitro expansion mediates the regression of metastatic melanoma $[20,25,26]$. $\mathrm{CD}^{+} \mathrm{PD} 1^{+} \mathrm{TILs}$ in the tumor microenvironment may be exactly the population that is being chronically exposed to relevant tumor antigens. In our study, we found that PD $1^{\text {hi }}$ in fresh CD8 ${ }^{+}$TILs predicted good prognosis in adjuvant chemotherapy plus TILs therapy group. We speculate that after culture more TILs recognize tumor cells in PD $1^{\text {hi }}$ patients. Therefore, PD $1^{\text {hi }}$ in adjuvant chemotherapy plus TILs therapy group is a good prognostic factor. In the future, selectively cultured PD1+ TILs may be optimal cells therapy in clinical applications and can yield higher antitumor reactivity.

At last, our study had some limitations. It was a single center retrospective clinical study and a prospective paired study is needed to confirm the efficacy of adjuvant chemotherapy combined with TILs therapy for osteosarcoma patients with a poor response to neoadjuvant chemotherapy. We only enrolled patients with a poor response to neoadjuvant chemotherapy in this study, which might underestimate the efficacy of TILs. More patients are needed for further analyzing the clinical benefit of TILs therapy for patients with osteosarcoma; however, our study was an exploration of treatment for osteosarcoma patients with poor response to neoadjuvant chemotherapy and provided some new insights into potential approaches for osteosarcoma patients.

\section{Conclusion}

We provided clinical evidence that combined adjuvant chemotherapy with TILs therapy improves the mDFS and mOS of osteosarcoma patients with a poor response to neoadjuvant chemotherapy. In the future, randomized multicenter studies involving combined adjuvant chemotherapy with immunotherapy are warranted.

\section{Future perspective}

Immunotherapies have emerged as an attractive treatment method and changed the therapeutic landscape for many cancer types. However, it is unclear whether osteosarcoma patients with a poor response to neoadjuvant chemotherapy can benefit from TILs therapy. We retrospectively investigate the efficacy of adjuvant chemotherapy plus TILs therapy in osteosarcoma patients with a poor response to neoadjuvant chemotherapy. Our results indicate that adjuvant chemotherapy plus TILs therapy improves the mDFS and mOS of osteosarcoma patients with a poor response to neoadjuvant chemotherapy. PD1 ${ }^{\text {hi }}$ in adjuvant chemotherapy plus TILs therapy group is a good prognostic factor. In the future, selectively cultured PD1 ${ }^{+}$TILs may be optimal cells therapy in clinical applications and can yield higher antitumor reactivity. Further studies are needed to confirm the efficacy of adjuvant chemotherapy combined with TILs therapy for osteosarcoma patients with a poor response to neoadjuvant chemotherapy. Additionally, more studies are also required to explore the underlying mechanism of the impact of TILs therapy in osteosarcoma patients and to precisely define 'patients' who are suitable for TILs therapy. 
Financial \& competing interests disclosure

The authors have no relevant affiliations or financial involvement with any organization or entity with a financial interest in or financial conflict with the subject matter or materials discussed in the manuscript. This includes employment, consultancies, honoraria, stock ownership or options, expert testimony, grants or patents received or pending, or royalties.

No writing assistance was utilized in the production of this manuscript.

Ethical conduct of research

This single-center retrospective clinical study was approved by the Ethics Committee at the Affiliated Luoyang Central Hospital of Zhengzhou University. All methods and procedures associated with this study were conducted in accordance with the Good Clinical Practice guidelines and accorded ethically with the principles of the Declaration of Helsinki and local laws. Institutional review board approval and data sharing agreements were obtained from all participating institutions. All data were anonymized.

Data availability statement

All data generated in the study are included in the present article and its supplementary information files.

\section{Open access}

This work is licensed under the Attribution-NonCommercial-NoDerivatives 4.0 Unported License. To view a copy of this license, visit http://creativecommons.org/licenses/by-nc-nd/4.0/

\section{Summary points}

- The identification of new methods of adjuvant therapy for osteosarcoma patients is urgently needed, especially patients with a poor response to neoadjuvant chemotherapy.

- The current recommendation for osteosarcoma patients with a poor response to neoadjuvant chemotherapy is chemotherapy alone.

- Modifying the dose intensity of chemotherapy agents or adding new chemotherapy agents have been unsuccessful in osteosarcoma patients with a poor response to neoadjuvant chemotherapy.

- The median disease-free survival (mDFs; 65.3 months) and median overall survival (mOS; 95.8 months) in the Group of adjuvant chemotherapy plus tumor-infiltrating lymphocytes (TILs) therapy were significantly prolonged than those in the Group of adjuvant chemotherapy (55.5 months for mDFS and 80.4 months for mOS). Univariate and multivariate analyses indicated that a greater number of TILs transfused was an independent prognostic factor for both mDFS and mOS.

- PD1 expression by $\mathrm{CD}^{+} \mathrm{CD} 8^{+}$TILs showed different prognostic effects between Groups of adjuvant chemotherapy plus TILs therapy and adjuvant chemotherapy. PD1 ${ }^{\text {hi }}$ is a poor prognostic factor in the Group of adjuvant chemotherapy, but a good prognostic factor in the Group of adjuvant chemotherapy plus TILs therapy.

- Adjuvant chemotherapy plus TILs therapy may prolong survival of patients with a poor response to neoadjuvant chemotherapy. In the future, randomized multicenter studies involving combined adjuvant chemotherapy with immunotherapy are warranted.

\section{References}

1. Siegel RL, Miller KD, Jemal A. Cancer statistics, 2019. CA Cancer J. Clin. 69(1), 7-34 (2019).

2. Mirabello L, Troisi RJ, Savage SA. International osteosarcoma incidence patterns in children and adolescents, middle ages and elderly persons. Int. J. Cancer 125(1), 229-234 (2009).

3. Mirabello L, Troisi RJ, Savage SA. Investing in workforce literacy pays: building employer commitment to workplace language, literacy and numeracy programs: [summary report]. Intern. J. Cancer 125(1), 229-234 (2011).

4. Grimer RJ, Bielack S, Flege S et al. Periosteal osteosarcoma - a European review of outcome. Eur. J. Cancer 41(18), $2806-2811$ (2005).

5. Anninga JK, Gelderblom H, Fiocco M et al. Chemotherapeutic adjuvant treatment for osteosarcoma: Where do we stand? Eur. J. Cancer 47(16), 2431-2445 (2011).

6. Cesari M, Alberghini M, Vanel D et al. Periosteal osteosarcoma. Cancer 117(8), 1731-1735 (2011).

7. Bacci G, Ferrari S, Tienghi A et al. A comparison of methods of loco-regional chemotherapy combined with systemic chemotherapy as neo-adjuvant treatment of osteosarcoma of the extremity. Eur. J. Surg. Oncol. 27(1), 98-104 (2001).

8. Winkler K, Beron G, Delling G et al. Neoadjuvant chemotherapy of osteosarcoma: results of a randomized cooperative trial (COSS-82) with salvage chemotherapy based on histological tumor response. J. Clin. Oncol. 6(2), 329-337 (1988). 
9. Marina NM, Smeland S, Bielack SS et al. Comparison of MAPIE versus MAP in patients with a poor response to preoperative chemotherapy for newly diagnosed high-grade osteosarcoma (EURAMOS-1): an open-label, international, randomised controlled trial. Lancet Oncol. 17(10), 1396-1408 (2016).

10. Provisor AJ, Ettinger LJ, Nachman JB et al. Treatment of nonmetastatic osteosarcoma of the extremity with preoperative and postoperative chemotherapy: a report from the Children's Cancer Group. J. Clin. Oncol. 15(1), 76-84 (1997).

11. Bacci G, Mercuri M, Longhi A et al. Grade of chemotherapy-induced necrosis as a predictor of local and systemic control in 881 patients with non-metastatic osteosarcoma of the extremities treated with neoadjuvant chemotherapy in a single institution. Eur. J. Cancer 41(14), 2079-2085 (2005).

12. Bernthal NM, Federman N, Eilber FR et al. Long-term results ( $>25$ years) of a randomized, prospective clinical trial evaluating chemotherapy in patients with high-grade, operable osteosarcoma. Cancer 118(23), 5888-5893 (2012).

13. Bacci G, Forni C, Longhi A et al. Long-term outcome for patients with non-metastatic Ewing's sarcoma treated with adjuvant and neoadjuvant chemotherapies. 402 patients treated at Rizzoli between 1972 and 1992. Eur. J. Cancer 40(1), 73-83 (2004).

14. Souhami RL, Craft AW, Van Der Eijken JW et al. Randomised trial of two regimens of chemotherapy in operable osteosarcoma: a study of the European Osteosarcoma Intergroup. Lancet 350(9082), 911-917 (1997).

15. Antman K, Crowley J, Balcerzak SP et al. A southwest oncology group and cancer-and leukemia group B phase II study of doxorubicin, dacarbazine, ifosfamide, and mesna in adults with advanced osteosarcoma, Ewing's sarcoma, and rhabdomyosarcoma. Cancer 82(7), 1288-1295 (1998).

16. Zalupski MM, Rankin C, Ryan JR et al. Adjuvant therapy of osteosarcoma - a Phase II trial: Southwest Oncology Group Study 9139. Cancer 100(4), 818-825 (2004).

17. Bielack SS, Hecker-Nolting S, Blattmann C, Kager L. Advances in the management of osteosarcoma. F1000Research 5, 2767 (2016).

18. Brahmer JR, Tykodi SS, Chow LQM et al. Safety and activity of anti-PD-L1 antibody in patients with advanced cancer. N. Engl. J. Med. 366(26), 2455-2465 (2012).

19. Liu L, Zhang W, Qi X et al. Randomized study of autologous cytokine-induced killer cell immunotherapy in metastatic renal carcinoma. Clin. Cancer Res. 18(6), 1751-1759 (2012).

20. Besser MJ, Shapira-Frommer R, Itzhaki $\mathrm{O}$ et al. Adoptive transfer of tumor-infiltrating lymphocytes in patients with metastatic melanoma: intent-to-treat analysis and efficacy after failure to prior immunotherapies. Clin. Cancer Res. 19(17), 4792-4800 (2013).

21. Chen CL, Pan QZ, Weng DS et al. Safety and activity of PD-1 blockade-activated DC-CIK cells in patients with advanced solid tumors. Oncoimmunology 7(4), e1417721 (2018).

22. Schell TD, Knowles BB, Tevethia SS. Sequential loss of cytotoxic T lymphocyte responses to simian virus 40 large $\mathrm{T}$ antigen epitopes in $\mathrm{t}$ antigen transgenic mice developing osteosarcomas. Cancer Res. 60(11), 3002-3012 (2000).

23. Lussier DM, O’Neill L, Nieves LM et al. Enhanced T-cell immunity to osteosarcoma through antibody blockade of PD-1/PD-L1 interactions. J. Immunother. 38(3), 96-106 (2015).

24. Ichino $\mathrm{Y}$, Ishikawa T. Cytolysis of autologous fresh osteosarcoma cells by human cytotoxic $\mathrm{T}$ lymphocytes propagated with $\mathrm{T}$ cell growth factor. Gann Japanese J. Cancer Res. 74(4), 584-594 (1983).

25. Rosenberg SA, Yannelli JR, Yang JC et al. Treatment of patients with metastatic melanoma with autologous tumor-infiltrating lymphocytes and interleukin 2. J. Natl Cancer Inst. 86(15), 1159-1166 (1994).

26. Dudley ME, Yang JC, Sherry R et al. Adoptive cell therapy for patients with metastatic melanoma: evaluation of intensive myeloablative chemoradiation preparative regimens. J. Clin. Oncol. 26(32), 5233-5239 (2008).

27. Théoleyre S, Mori K, Cherrier B et al. Phenotypic and functional analysis of lymphocytes infiltrating osteolytic tumors: Use as a possible therapeutic approach of osteosarcoma. BMC Cancer doi: 10.1186/1471-2407-5-123 (2005).

28. Li W, Xu L, Wang Y, Zhao L, Kellner DB, Gao Q. Efficacy of tumor-infiltrating lymphocytes combined with IFN- $\alpha$ in Chinese resected stage III malignant melanoma. J. Immunol. Res. 2017, 1092507 (2017).

29. Berner K, Hall KS, Monge OR, Weedon-Fekjær H, Zaikova O, Bruland ØS. Prognostic factors and treatment results of high-grade osteosarcoma in Norway: a scope beyond the "classical" patient. Sarcoma 2015, 516843 (2015).

30. Meyers PA, Schwartz CL, Krailo MD et al. Osteosarcoma: the addition of muramyl tripeptide to chemotherapy improves overall survival - A report from the children's oncology group. J. Clin. Oncol. 26(4), 633-638 (2008).

31. Yannelli JR, Hyatt C, McConnell S et al. Growth of tumor-infiltrating lymphocytes from human solid cancers: summary of a 5-year experience. Int. J. Cancer 65(4), 413-421 (1996).

32. Stevanović S, Draper LM, Langhan MM et al. Complete regression of metastatic cervical cancer after treatment with human papillomavirus-targeted tumor-infiltrating T cells. J. Clin. Oncol. 33(14), 1543-1550 (2015).

33. Andersen R, Westergaard MCW, Kjeldsen JW et al. T-cell responses in the microenvironment of primary renal cell carcinoma-implications for adoptive cell therapy. Cancer Immunol. Res. 6(2), 222-235 (2018).

34. Stevanovic S, Helman SR, Wunderlich JR et al. A Phase II study of tumor-infiltrating lymphocyte therapy for human papillomavirus-associated epithelial cancers. Clin. Cancer Res. 25(5), 1486-1493 (2019). 
35. Rainusso N, Brawley VS, Ghazi A et al. Immunotherapy targeting HER2 with genetically modified $\mathrm{T}$ cells eliminates tumor-initiating cells in osteosarcoma. Cancer Gene Ther. 19(3), 212-217 (2012).

36. Zhang L, Dermawan K, Jin M et al. Differential impairment of regulatory $\mathrm{T}$ cells rather than effector $\mathrm{T}$ cells by paclitaxel-based chemotherapy. Clin. Immunol. 129(2), 219-229 (2008).

37. Kodumudi KN, Woan K, Gilvary DL, Sahakian E, Wei S, Djeu JY. A novel chemoimmunomodulating property of docetaxel: Suppression of myeloid-derived suppressor cells in tumor bearers. Clin. Cancer Res. 16(18), 4583-4594 (2010).

38. Gattinoni L, Finkelstein SE, Klebanoff CA et al. Removal of homeostatic cytokine sinks by lymphodepletion enhances the efficacy of adoptively transferred tumor-specific CD8+ T cells. J. Exp. Med. 202(7), 907-912 (2005).

39. Bielack SS, Kempf-Bielack B, Delling G et al. Prognostic factors in high-grade osteosarcoma of the extremities or trunk: an analysis of 1,702 patients treated on neoadjuvant cooperative osteosarcoma study group protocols. J. Clin. Oncol. 20(3), 776-790 (2002).

40. Whelan JS, Jinks RC, McTiernan A et al. Survival from high-grade localised extremity osteosarcoma: combined results and prognostic factors from three European osteosarcoma intergroup randomised controlled trials. Ann. Oncol. 23(6), 1607-1616 (2012).

41. Bertrand TE, Cruz A, Binitie O, Cheong D, Letson GD. Do surgical margins affect local recurrence and survival in extremity, nonmetastatic, high-grade osteosarcoma? Clin. Orthop. Relat. Res. 474(3), 677-683 (2016).

42. Ahmadzadeh M, Johnson LA, Heemskerk B et al. Tumor antigen-specific CD8 T cells infiltrating the tumor express high levels of PD-1 and are functionally impaired. Blood 114(8), 1537-1544 (2009).

43. Inozume T, Hanada KI, Wang Q et al. Selection of CD8++PD-1+ lymphocytes in fresh human melanomas enriches for tumor-reactive T cells. J. Immunother. 33(9), 956- 64 (2010). 